\title{
Validation of a novel prognostic index: BMS-Score for patients with brain metastases of small cell lung cancer
}

\author{
Ru Hou ${ }^{1}$, Hongwei $\mathrm{Li}^{2}$, Jianzhong $\mathrm{Cao}^{2}$, Xin Song ${ }^{2}$, Xiaqin Zhang ${ }^{2}$, Weili Wang ${ }^{2}$ \\ ${ }^{1}$ Shanxi Medical University, Taiyuan, China; ${ }^{2}$ Department of Radiotherapy, Shanxi Province Cancer Hospital and Affiliated Cancer Hospital of \\ Shanxi Medical University, Taiyuan, China \\ Contributions: (I) Conception and design: H Li; (II) Administrative support: H Li; (III) Provision of study materials or patients: H Li; (IV) Collection \\ and assembly of data: R Hou, X Song, X Zhang; (V) Data analysis and interpretation: R Hou, J Cao, W Wang; (VI) Manuscript writing: All authors; \\ (VII) Final approval of manuscript: All authors. \\ Correspondence to: Hongwei Li, MD. Department of Radiotherapy, Shanxi Province Cancer Hospital and Affiliated Cancer Hospital of Shanxi Medical \\ University, Taiyuan, China. Email: 3420010@163.com.
}

Background A new disease-specific prognostic score (Disease-specific Prognostic Score for Patients With Brain Metastases From Small-cell Lung Cancer termed BMS-Score) was published to clarify the prognosis of patients with brain metastasis (BM) of small cell lung cancer (SCLC) treated with whole brain radiotherapy (WBRT). The purpose of the present study was to validate the prognostic value of the newly proposed BMSScore through comparison with three other previously published prognostic indices.

Methods: In total, 451 patients with BM of SCLC treated with WBRT at the Shanxi Province Cancer Hospital from January 2010 to December 2019 were included. The clinical characteristics of all patients were recorded and follow-up was through April 2020. Overall survival (OS) was calculated by KaplanMeier analysis, and univariate and multivariate analyses were used to calculate the prognostic cofactors. The concordance index (C-index) was used to assess the prognostic value of the following four prognostic systems: recursive partitioning analysis (RPA), diagnosis-specific graded prognostic assessment (DS-GPA), basic score for brain metastases (BSBM), and the newly proposed BMS-Score.

Results: The independent factors affecting the prognosis of SCLC patients with BM included the Karnofsky performance score (KPS), number of brain metastases, extracranial metastases (ECM) state, and whether treatment had been received before BM. RPA, BSBM, DS-GPA, and BMS-Score log-rank test P values were all less than 0.001 among each group $(\mathrm{P}<0.001)$. The $\mathrm{C}$-indices of the four groups were 0.554 , $0.584,0.588$, and 0.643 , respectively.

Conclusions: The four prognostic scoring systems exhibited medium predictive value for SCLC. The BMS-Score had the best applicability compared with the other three prognosis indices.

Keywords: Prognostic index; small cell lung cancer (SCLC); brain metastases; whole brain radiotherapy (WBRT); survival

Submitted Aug 14, 2020. Accepted for publication Nov 14, 2020.

doi: 10.21037/apm-20-1819

View this article at: http://dx.doi.org/10.21037/apm-20-1819

\section{Introduction}

Lung cancer has the highest morbidity and mortality of all malignancies worldwide (1). Small cell lung cancer (SCLC) accounts for approximately $15-20 \%$ of lung cancers (2). SCLC patients have a particularly high propensity to develop brain metastases (BMs), with 10-20\% SCLC patients harboring brain metastasis at initial diagnosis and 50-80\% developing brain metastasis during the treatment process (3-5). Due to the propensity for multiple brain metastases (2), the median survival times (MSTs) of patients with BMs is approximately 1-2 months without 
treatment, while the standard treatment with WBRT can improve survival to 3-6 months (6-8). WBRT is the firstline treatment, with a recommended dose of 30 Gy in 10 fractions or $40 \mathrm{~Gy}$ in 20 fractions $(9,10)$. However, most patients exhibit significant heterogeneity after receiving WBRT (11). To individualize patient treatment, stratifiedspecific prognostic indices are needed for this cohort.

Previous studies focused mainly on BMs, and specific studies on the prognosis in SCLC with BM are few. Several prognostic index systems were previously published including 1997, Gaspar et al. created recursive partitioning analysis (RPA), in 2004, basic score for brain metastases (BSBM) proposed by Lorenzoni et al. and Sperduto et al. Constructed the revised diagnosis-specific graded prognostic assessment (DS-GPA) in 2010; however, these index studies enrolled a small number of SCLC patients (12-14). In 2018, Bernhardt et al. developed a new prognostic evaluation system specifically for SCLC BM treated with WBRT: the BMS-Score, this is the first prognostic index for small cell lung cancer with brain metastases and it includes three factors: KPS, synchronous BM or metachronous BM, and ECM state (15). These three factors are assigned a different weight points: 2 points for KPS $\geq 70,1$ point for synchronous BM, 1 point for ECM state stable, the scores of each patient was the sum of points in each factor. The purpose of the present study is to validate the prognostic value of the newly proposed BMS-Score by comparing it with the RPA, BSBM, and DS-GPA prognostic indices.

We present the following article in accordance with the MDAR reporting checklist (available at http://dx.doi. org/10.21037/apm-20-1819).

\section{Methods}

\section{Study inclusion criteria}

The present study was carried out at a single institution, the Shanxi Province Cancer Hospital. We retrospectively collected the records of patients $(\mathrm{n}=801)$ with histologically confirmed SCLC with BM from January 2010 to December 2019, BMs newly diagnosed within 3 months and confirmed by enforced computed tomography (CT) and/or magnetic resonance imaging (MRI), and those treated with WBRT. Patients treated with prior cranial radiotherapy (PCI, $\mathrm{n}=43)$, only palliative chemotherapy $(\mathrm{n}=301)$, stereotactic radiosurgery (SRS, $n=3$ ), or prior surgery $(n=3)$, were excluded. All of the patients had complete and detailed clinical data and dates of death or follow-up examinations.
Ultimately, 451 patients were eligible for analysis. Patient characteristics are listed in Table 1.

WBRT was performed in all patients. Of these, 294 patients were treated with 30 Gy in 10 fractions, 134 patients were treated with 40 Gy in 20 fractions, and 23 patients did not complete the full treatment course due to deterioration of their general condition. Furthermore, $33.7 \%$ of the patients received an additional dose (WBRT-add) of 10-20 Gy in 2 doses/day depending upon the individual BM. All patients received appropriate chemoradiotherapy for their primary disease and extracranial metastases (ECM) according to their condition.

All procedures performed in this study involving human participants were in accordance with the Declaration of Helsinki (as revised in 2013). Individual consent for this retrospective analysis was waived. This study was approved by the Shanxi Provincial Cancer Hospital ethics committee.

\section{Analysis of prognostic factors and stratification}

In this study, clinical characteristics included the following: gender, age, Karnofsky performance score (KPS) at the time of $\mathrm{BM}$ diagnosis, time of $\mathrm{BM}$, number of $\mathrm{BMs}$, smoking history, synchronous BM, metachronous BM, with or without ECM, ECM state, control of the primary tumor, and whether the patient had received first-line chemotherapy and radiation prior to BM. Synchronous $\mathrm{BM}$ was defined at the time of initial diagnosis of the primary tumor, including BMs that were newly diagnosed within 3 months. ECM state at the time of diagnosis of brain metastasis $(\mathrm{BM})$ [stability or progress were measured according to the Response Evaluation Criteria in Solid Tumors (RECIST) standard based on the latest CT scan] (16). Synchronous BM was defined as progressive disease, and those without ECM were considered stable. Stratification was performed according to the RPA, BSBM, DS-GPA, and BMS-Score indices.

\section{Statistics}

The endpoint of the study was overall survival (OS), which was estimated from the first day of discovery of BMs to the time of death or last date of follow-up. Kaplan-Meier survival curves were created to estimate OS, and the logrank test was used for univariate analysis to compare the difference among the subgroups. Multivariate Cox proportional-hazard ratios were applied to assess the independent factors. $\mathrm{P}<0.05$ was considered statistically 
Table 1 Characteristics of 451 SCLC BM patients treated with WBRT

\begin{tabular}{|c|c|c|c|}
\hline Variables & MSTs (months) & Number of patients & $\%$ \\
\hline \multicolumn{4}{|l|}{ Gender } \\
\hline Female & 13.43 & 82 & 18.20 \\
\hline Male & 11.40 & 369 & 81.80 \\
\hline \multicolumn{4}{|l|}{ Age group } \\
\hline$<50$ & 13.43 & 79 & 17.50 \\
\hline $50-60$ & 12.30 & 188 & 41.70 \\
\hline$>60$ & 10.30 & 184 & 40.80 \\
\hline \multicolumn{4}{|l|}{ KPS group } \\
\hline$<70$ & 6.63 & 62 & 13.70 \\
\hline $70-80$ & 12.60 & 280 & 62.10 \\
\hline $90-100$ & 13.90 & 109 & 24.20 \\
\hline \multicolumn{4}{|l|}{ No. BM group } \\
\hline 1 & 14.77 & 146 & 32.40 \\
\hline 2 & 14.83 & 51 & 11.30 \\
\hline 3 & 9.90 & 6 & 1.30 \\
\hline Multiple & 9.70 & 239 & 53.00 \\
\hline Unknown & 12.93 & 9 & 2.00 \\
\hline \multicolumn{4}{|l|}{ Smoking history } \\
\hline No & 12.70 & 120 & 26.60 \\
\hline Yes & 11.80 & 331 & 73.40 \\
\hline \multicolumn{4}{|c|}{ Synchronous/metachronous BM } \\
\hline Synchronous & 12.93 & 116 & 25.70 \\
\hline Metachronous & 11.47 & 335 & 74.30 \\
\hline \multicolumn{4}{|l|}{$\mathrm{ECM}$} \\
\hline No & 16.90 & 58 & 12.90 \\
\hline Yes & 11.00 & 393 & 87.10 \\
\hline \multicolumn{4}{|l|}{ ECM state } \\
\hline Stable & 17.00 & 160 & 35.50 \\
\hline Progress & 9.90 & 291 & 64.50 \\
\hline \multicolumn{4}{|c|}{ Control of primary tumor } \\
\hline Progress & 10.87 & 230 & 51.00 \\
\hline Stable & 13.07 & 221 & 49.00 \\
\hline \multicolumn{4}{|c|}{ Treatment before BM } \\
\hline No & 13.07 & 117 & 23.90 \\
\hline Yes & 11.37 & 343 & 76.10 \\
\hline Total & 11.87 & 451 & 100 \\
\hline
\end{tabular}

SCLC, small cell lung cancer; WBRT, whole brain radiotherapy; KPS, Karnofsky performance score; No. BM, number of brain metastases; BM, brain metastases; ECM, extracranial metastases; MSTs, median survival times. significant. The concordance index (C-index) was used to compare the predictive values of the four existing systems. Generally, a C-index greater than 0.50 is statistically significant, with values closer to 1 indicating greater accuracy. All of the analyses were performed using SPSS 26.0 (except for the C-index, which was calculated using $\mathrm{R}$ version 3.6.3 and R Studio-1.2.5033).

\section{Results}

\section{Patient characteristics}

A total of 451 patients with BMs were eligible for this study. Most of the patients (81.8\%) were male, $82.5 \%$ were older than 50 years, $73.4 \%$ had a history of smoking, and $86.3 \%$ were in good general condition (KPS $\geq 70$ ). Overall, $25.7 \%$ of BMs were identified as synchronous with the primary tumor and $53.0 \%$ of patients had multiple BMs. Of the 451 included patients, 393 (87.1\%) had BMs occurring simultaneously with extracranial lesions, with $64.5 \%$ of ECMs progressing and less than half of the patients (49.0\%) with BMs having stable primary tumors. Most of the patients $(76.1 \%)$ received first-line chemotherapy and radiation for primary lung lesions before BMs. Details are shown in Table 1.

\section{OS and prognoses}

At the last follow-up date, a total of 340 patients had died, with an OS maturity rate of $75.4 \%$. The MST for the whole series was 11.87 months [95\% confidence interval (CI), 10.611-13.129], with 1-, 2-, and 3-year survival rates of $49.4 \%, 21.5 \%$, and $12.1 \%$, respectively. The prognostic factors that influenced survival in the univariate analysis were gender, age, KPS, number of BMs, smoking history, with or without ECM, ECM state, and control of the primary tumor. Multivariable analysis showed that a higher KPS (KPS $\geq 70$ ), fewer number of BMs, stable ECM, and receiving treatment before $\mathrm{BM}$ were statistically significant survival factors (Table 2).

\section{Prognostic indices}

A comparison of the prognostic value of the four indices is shown in Table 3 and Figure 1. In the RPA, BSBM, DSGPA, and BMS-Score indices, survival curves demonstrated excellent separation among each group (log-rank test, $\mathrm{P}<0.001)$. According to RPA, most of the patients $(82.5 \%)$ 
Table 2 Results of the univariate and multivariate analysis of cofactors associated with overall survival

\begin{tabular}{lcccc}
\hline \multirow{2}{*}{ Variables } & Univariate & \multicolumn{4}{c}{ Multivariate analysis } \\
\cline { 3 - 5 } analysis (P) & Risk ratio & $95.0 \% \mathrm{Cl}$ & $\mathrm{P}$ \\
\hline Gender & 0.005 & - & - & - \\
Age group & 0.042 & - & - & - \\
KPS group & $<0.001$ & 0.463 & $0.339-0.633<0.001$ \\
No. BM group & $<0.001$ & 1.124 & $1.039-1.216$ & 0.004 \\
Smoking history & 0.048 & - & - & - \\
Synchronous BM & 0.917 & - & - & - \\
ECM & 0.001 & - & - & - \\
ECM state & $<0.001$ & 2.351 & $1.823-3.032<0.001$ \\
Control of primary & 0.003 & - & - & - \\
tumor & & & & \\
Treatment before & 0.628 & 1.511 & $1.162-1.965$ & 0.002 \\
BM & & & & \\
\hline
\end{tabular}

KPS, Karnofsky performance score; No. BM, number of brain metastases; BM, brain metastases; ECM, extracranial metastases.

were in class II, $4 \%$ were in class I, and $13.5 \%$ were in class III. We found that MSTs following WBRT were 16.9 months in class I, 12.83 months in class II, and 5.93 months in class III. For the BSBM group, the MST of the zero-point group was 7.47 months, 12.7 months for the one-point group, 13.63 months for the two-point group, and 17.43 months for the three-point group. The MSTs for all of the DS-GPA groups were 9.07, 13.6, 14.9, and 17.43 months, respectively. The BMS-Score revealed that the MST was 5.7 months in group one (0-1 points), 9.4 months in group two ( 2 points), and 16.2 months in group three (3-4 points). The number of patients with a BMS-Score of 0 to $1,2,3$, and 4 points was $57,167,224$, and 3 , respectively. In the 4-points group, only two patients had an outcome, so those with three and four points were merged into Group 3.

Patients in the worst groups of the four systems (RPA: class III, BSBM: zero points, DS-GPA: 0-1 points, and BMS-Score: Group One) had MSTs of 5.93, 7.47, 9.07, and 5.7 months, respectively. In addition, MSTs in the best groups of these systems (RPA: class I, BSBM: three points, DS-GPA: 3.5-4.0 points, and BMS-Score: Group Three) were 16.9, $17.43,17.43$, and 16.2 months, respectively. Moreover, due to a deterioration of general condition, 23 patients did not complete the full course of WBRT. In this subgroup, the MST was only 4.4 months, and all of the four prognostic grading
Table 3 Log-rank analyses of four scoring systems

\begin{tabular}{|c|c|c|c|c|c|}
\hline \multirow{2}{*}{ Group } & \multirow{2}{*}{ Patients } & \multirow{2}{*}{$\begin{array}{c}\text { MSTs } \\
\text { (months) }\end{array}$} & \multicolumn{2}{|c|}{$95 \% \mathrm{Cl}$} & \multirow{2}{*}{ - Log-rank P } \\
\hline & & & Low & High & \\
\hline RPA & & & & & $<0.001$ \\
\hline I class & 18 & 16.900 & 11.686 & 22.114 & \\
\hline II class & 372 & 12.830 & 11.577 & 14.083 & \\
\hline III class & 61 & 5.930 & 3.709 & 8.151 & \\
\hline BSBM & & & & & $<0.001$ \\
\hline Zero-point & 83 & 7.470 & 5.839 & 9.101 & \\
\hline One-point & 181 & 12.700 & 10.741 & 14.659 & \\
\hline Two-point & 164 & 13.630 & 10.204 & 17.056 & \\
\hline Three-point & 23 & 17.430 & 5.498 & 29.362 & \\
\hline DS-GPA & & & & & $<0.001$ \\
\hline $0-1.0$ & 186 & 9.070 & 7.751 & 10.389 & \\
\hline $1.5-2.0$ & 191 & 13.600 & 11.906 & 15.294 & \\
\hline $2.5-3.0$ & 70 & 14.900 & 11.687 & 18.113 & \\
\hline $3.5-4.0$ & 4 & 17.430 & 14.293 & 20.567 & \\
\hline BSM-Score & & & & & $<0.001$ \\
\hline Group one & 57 & 5.700 & 3.942 & 7.458 & \\
\hline Group two & 167 & 9.400 & 6.604 & 12.196 & \\
\hline Group three & 227 & 16.200 & 14.466 & 17.934 & \\
\hline Total & 451 & 11.870 & 10.611 & 13.129 & \\
\hline
\end{tabular}

The median survival times of the four prognostic index and log rank-test were all less than 0.001 among each group $(\mathrm{P}<0.001)$. MSTs, median survival times; RPA, recursive partitioning analysis; DS-GPA, diagnosis-specific graded prognostic assessment; BSBM, basic score for brain metastases.

systems were able to predict poor survival in this subgroup.

The C-index was calculated to compare the predictive values of each scoring system. The C-index values for RPA, BSBM, DS-GPA, and BMS-Score were 0.554, 0.584, 0.588 , and 0.643 , respectively. These results suggested that the four scoring systems had medium predictive value for SCLC BMs treated with WBRT. The BMS-Score exhibited the best applicability (Table 4).

\section{Discussion}

Patients suffering from SCLC with BM have a poor prognosis following treatment due to the heterogeneous nature of the disease (11). The reported median survival 

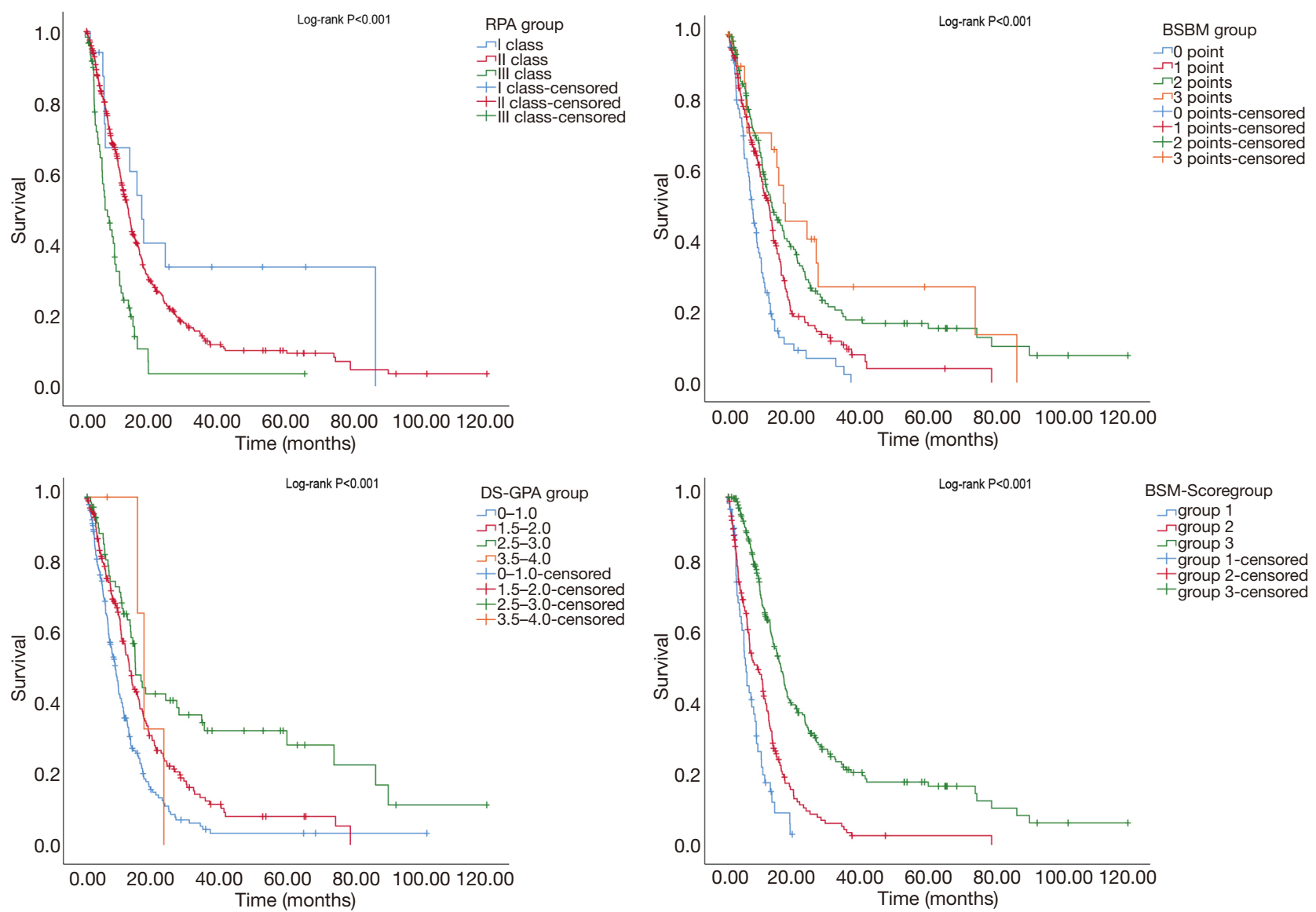

Figure 1 Kaplan-Meier curves showing SCLC BM patient survival using the RPA, BSBM, DS-GPA, and BMS-Score prognostic indices, respectively. SCLC, small cell lung cancer; BM, brain metastasis; RPA, recursive partitioning analysis; BSBM, basic score for brain metastases; DS-GPA, diagnosis-specific graded prognostic assessment.

Table 4 The predictive value of the four prognostic systems (C-index)

\begin{tabular}{lccc}
\hline Score & Group & Number of patients & C-index \\
\hline RPA & I/II/III & $18 / 372 / 61$ & 0.544 \\
BSBM & Zero/one/two/three & $83 / 181 / 164 / 23$ & 0.584 \\
DS-GPA & $0-1 / 1.5-2 / 2.5-3 / 3.5-4$ & $186 / 191 / 70 / 4$ & 0.588 \\
BMS- & One/two/three & $57 / 167 / 227$ & 0.643 \\
Score & & & \\
\hline
\end{tabular}

RPA, recursive partitioning analysis; DS-GPA, diagnosis-specific graded prognostic assessment; BSBM, basic score for brain metastases.

for these patients ranges from 1 to 14 months $(3,10)$. An accurate stratified benefit-risk evaluation based on prognosis is generally a clinically efficacious tool for making individualized and precise treatment decisions (4). Recently, Bernhardt et al. developed a new prognostic evaluation system specifically for SCLC BM called the BMS-Score (15). In this study, we initially enrolled 451 patients to validate this novel index. Through calculating the $\mathrm{C}$-index value, we found the BMS-score index to have medium predictive ability, although it did exhibit better predictive value compared with the previously published RPA, BSBM, and DS-GPA indices. However, we identified some drawbacks to this predictive system that require further revision.

The original work for the prognostic study only went back to 1997, Gaspar et al. collected the data from 1,200 BM patients with various cancer types in three WBRT clinical trials by the Radiation Therapy Oncology Groups (RTOG) between 1979 and 1993, and published the first prognostic system (RPA), which included four factors: age, 
KPS, control of the primary tumor, and with or without ECM (12). Among all of these BM patients, only $4 \%$ $(\mathrm{n}=51)$ had SCLC. In 2004, the BSBM score system was proposed by Lorenzoni et al. based on $110 \mathrm{BM}$ patients who were divided into three levels based on the sum of the score (zero or one points) from three main variables: KPS, primary tumor control, and presence or absence of ECM (13). Since these indices included incomplete independent prognostic factors, Sperduto et al. developed the GPA system in 2008 based on 1,960 patients from four RTOG (17). Furthermore, to specify the prognostic index for different cancer types, Sperduto et al. constructed the updated DS-GPA, which was based on 5,067 patients from 11 institutions between 1985 and 2007. This system has been widely validated and includes four factors (age, KPS, number of BMs, and presence or absence of ECM) (14). However, SCLC BM patients were grouped with nonSCLC (NSCLC) patients, and only a small number of SCLC patients were included.

There are two SCLC BM-specific prognostic score systems: the Rades-SCLC and the BMS-Score $(15,18)$. In 2013, Rades et al. proposed the Rades-SCLC scoring system, which was based on 117 SCLC BM patients treated with WBRT. The score of each independent prognostic factor (including KPS, number of BMs, and ECM) acquired points based on their weight on prognosis, with the sum of three factors in each patient constituting his/her final score. Patients were then grouped into three prognostic stratifications according to their total points (18). However, this system is complex and has limited predictive ability, and is thus not often used in clinical practice $(19,20)$. In 2018, Bernhardt et al. proposed a novel index called the BMS-Score, which consists of only three factors: KPS, synchronous BM or metachronous BM, and ECM state (15). This novel SCLC BM-specific prognostic index was easier to be used compared with the previous ones, even by simple clinical examinations, a relatively accurate assessment for the patients' prognosis can be made from three easierto-measure variables. Then patients will be divided into different prognostic categories.

To further validate the BMS-Score system and confirm its prognostic value for SCLC BM patients treated with WBRT, we retrospectively enrolled 451 patients and analyzed the predictive capability of the four prognostic scoring systems. The C-index values for RPA, BSBM, DSGPA, and BMS-Score were 0.554, 0.584, 0.588, and 0.643, respectively, indicating that they have medium predictive value. Furthermore, the BMS-Score exhibited the best predictive ability, suggesting its potential prognostic value. However, it was derived from a relatively small sample size $(\mathrm{n}=221)$, and the only treatment option was WBRT. In fact, there are numerous treatment modalities for SCLC $\mathrm{BM}$ in routine clinical practice. For example, In recent years, mounting evidence has shown that immunotherapy may be a promising method for this cohort of patients .IMpower-133 study shows that the first-line randomized trial for Extensive stage small cell lung cancer comparing etoposide + carboplatin + placebo with etoposide + carboplatin + atezolizumab, the median overall survival was 12.3 months in the atezolizumab group and 10.3 months in the placebo group (hazard ratio for death, 0.70); another research named CASPIAN study also showed positive results. Comparing with the chemotherapy alone, the median survival of durvalumab combined chemotherapy group was improved 2-7 months. However, efficacy in the subgroup of brain metastases further reform is needed (21-23). In the era of multidisciplinary treatment, the optimal prognostic index should consider all therapeutic options. Additionally, a concern regarding WBRT is the long-term risk of cognitive decline $(24,25)$. This may also have an effect on survival and quality of life. Thus, to further optimize this index, such factors should be incorporated into future updates.

In the present study, the MST was 11.87 months, which is relatively better than in previous reports $(14,20)$. This was mainly due to the retrospective design and an inherent selection bias. Also, most patients in this study presented with a favorable performance state, with $86.3 \%$ of KPS scores greater than 70 points. Moreover, the longer survival might be related to the fact that BMs tend to be detected early by more sensitive imaging systems such as MRI, and patients were treated in the recent decade. In the present study, the main independent predictive factors associated with OS were KPS, number of BMs, ECM state, and receipt of treatment before $\mathrm{BM}$, which is consistent with previous studies $(14,15,18,26)$. However, in contrast to Bernhardt et al. (15), there was no significant statistical difference between synchronous and metachronous BM. According to national guidelines, SCLC with BM should be treated with WBRT at a recommended dose of $30 \mathrm{~Gy}$ in 10 fractions, supported by diffuse intracranial disease features $(27,28)$. In our institution, 30 Gy in 10 daily fractions is generally adopted, with some patients being treated with a 40 Gy dose in 20 fractions due to edema present around large tumors. Borgelt et al. compared the first two randomized national Phase III trials carried out by the RTOG and showed that differing radiation doses and 
fractionation schemes did not make a substantial difference to OS $(29,30)$. Thus, the two dose patterns did not affect the experimental results in this study.

In conclusion, this study showed that the four prognostic scoring systems exhibit medium predictive value for SCLC prognosis. Compared with the RPA, BSBM, and DS-GPA prognosis indices, the BMS-Score had the best applicability for SCLC BM treated with WBRT. More importantly, it provides a basis for further studies of BM in SCLC. In future, it is necessary to update the prognostic index via a large multicenter trial, and more potential independent prognostic factors and multi-treatment options should be considered.

\section{Acknowledgments}

We thank LetPub (www.letpub.com) for its linguistic assistance during the preparation of this manuscript. Funding: Natural Science Foundation of Shanxi province in China (201701D12116).

\section{Footnote}

Reporting Checklist: The authors have completed the MDAR reporting checklist. Available at http://dx.doi.org/10.21037/ apm-20-1819

Data Sharing Statement: Available at http://dx.doi. org/10.21037/apm-20-1819

Conflicts of Interest: All authors have completed the ICMJE uniform disclosure form (available at http://dx.doi. org/10.21037/apm-20-1819). The authors have no conflicts of interest to declare.

Ethical Statement: The authors are accountable for all aspects of the work in ensuring that questions related to the accuracy or integrity of any part of the work are appropriately investigated and resolved. All procedures performed in this study involving human participants were in accordance with the Declaration of Helsinki (as revised in 2013). Individual consent for this retrospective analysis was waived. This study was approved by the Shanxi Provincial Cancer Hospital ethics committee.

Open Access Statement: This is an Open Access article distributed in accordance with the Creative Commons Attribution-NonCommercial-NoDerivs 4.0 International
License (CC BY-NC-ND 4.0), which permits the noncommercial replication and distribution of the article with the strict proviso that no changes or edits are made and the original work is properly cited (including links to both the formal publication through the relevant DOI and the license). See: https://creativecommons.org/licenses/by-nc-nd/4.0/.

\section{References}

1. Bray F, Ferlay J, Soerjomataram I, et al. Global cancer statistics 2018: GLOBOCAN estimates of incidence and mortality worldwide for 36 cancers in 185 countries. CA Cancer J Clin 2018;68:394-424.

2. Nayak L, Lee EQ, Wen PY. Epidemiology of brain metastases. Curr Oncol Rep 2012;14:48-54.

3. Quan AL, Videtic GMM, Suh JH. Brain metastases in small cell lung cancer. Oncology (Williston Park) 2004;18:961-72; discussion 974, 979-80, 987.

4. Steindl A, Schlieter F, Klikovits T, et al. Prognostic assessment in patients with newly diagnosed small cell lung cancer brain metastases: results from a real-life cohort. J Neurooncol 2019;145:85-95.

5. Rades D, Nguyen T, Janssen S, et al. Development of a multivariable prediction model to estimate the remaining lifespan of elderly patients with cerebral metastases from small-cell lung cancer. Transl Lung Cancer Res 2020;9:1433-40.

6. Eichler AF, Kahle KT, Wang DL, et al. EGFR mutation status and survival after diagnosis of brain metastasis in nonsmall cell lung cancer. Neuro Oncol 2010;12:1193-9.

7. Magnuson WJ, Yeung JT, Guillod PD, et al. Impact of Deferring Radiation Therapy in Patients With Epidermal Growth Factor Receptor-Mutant Non-Small Cell Lung Cancer Who Develop Brain Metastases. Int J Radiat Oncol Biol Phys 2016;95:673-9.

8. Gaspar LE, Mehta MP, Patchell RA, et al. The role of whole brain radiation therapy in the management of newly diagnosed brain metastases: a systematic review and evidence-based clinical practice guideline. J Neurooncol 2010;96:17-32.

9. Bernhardt D, Adeberg S, Bozorgmehr F, et al. Outcome and prognostic factors in single brain metastases from small-cell lung cancer. Strahlenther Onkol 2018;194:98-106.

10. National Comprehensive Cancer Network (NCCN) Clinical Practice Guidelines in Oncology. Small Cell Lung Cancer. Version 1.2018., 2017.

11. Li H, Lian J, Jin H, et al. Assessment of prognostic scores of brain metastases from lung adenocarcinoma with EGFR 
mutations. J Neurooncol 2017;133:129-35.

12. Gaspar L, Scott C, Rotman M, et al. Recursive partitioning analysis (RPA) of prognostic factors 316 in three Radiation Therapy Oncology Group (RTOG) brain metastases trials. Int J Radiat Oncol Biol Phys 1997;37:745-51.

13. Lorenzoni J, Devriendt D, Massager N, et al. Radiosurgery for treatment of brain metastases: estimation of patient eligibility using three stratification systems. Int J Radiat Oncol Biol Phys 2004;60:218-24

14. Sperduto PW, Chao ST, Sneed PK, et al. Diagnosis specific prognostic factors, indexes, and treatment outcomes for patients with newly diagnosed brain metastases: a multiinstitutional analysis of 4,259 patients. Int J Radiat Oncol Biol Phys 2010;77:655-61.

15. Bernhardt D, König L, Aufderstrasse S, et al. Generation of a New Disease-specific Prognostic Score for Patients With Brain Metastases From Small-cell Lung Cancer Treated With Whole Brain Radiotherapy (BMS-Score) and Validation of Two Other Indices. Clin Lung Cancer 2018;19:340-5.

16. Eisenhauer EA, Therasse P, Bogaerts J, et al. New response evaluation criteria in solid tumors: revised RECIST guideline (version 1.1). Eur J Cancer 2009;45:228-47.

17. Sperduto PW, Berkey B, Gaspar LE, et al. A new prognostic index and comparison to three other indices for patients with brain metastases: an analysis of 1,960 patients in the RTOG database. Int J Radiat Oncol Biol Phys 2008;70:510-4.

18. Rades D, Dziggel L, Segedin B, et al. The first survival score for patients with brain metastases from small cell lung cancer (SCLC). Clin Neurol Neurosurg 2013;115:2029-32.

19. Rades D, Dziggel L, Nagy V, et al. A new survival score for patients with brain metastases who received wholebrain radiotherapy (WBRT) alone. Radiother Oncol 2013;108:123-7.

20. Rades D, Hansen H, Janssen S, et al. Comparison of diagnosis-specific survival scores for patients with smallcell lung cancer irradiated for brain metastases. Cancers (Basel) 2019;11:233.

21. Horn L, Mansfield AS, Szczęsna A, et al. First-Line Atezolizumab plus Chemotherapy in Extensive-Stage

Cite this article as: Hou R, Li H, Cao J, Song X, Zhang X, Wang W. Validation of a novel prognostic index: BMS-Score for patients with brain metastases of small cell lung cancer. Ann Palliat Med 2021;10(1):29-36. doi: 10.21037/apm-20-1819
Small-Cell Lung Cancer. N Engl J Med 2018;379:2220-9.

22. Nishio M, Sugawara S, Atagi S, et al. Subgroup Analysis of Japanese Patients in a Phase III Study of Atezolizumab in Extensive-stage Small-cell Lung Cancer (IMpower133). Clin Lung Cancer 2019;20:469-476.e1.

23. Paz-Ares L, Dvorkin M, Chen Y, et al. Durvalumab plus platinum-etoposide versus platinum-etoposide in firstline treatment of extensive-stage small-cell lung cancer (CASPIAN): a randomised, controlled, open-label, phase 3 trial. Lancet 2019;394:1929-39.

24. Brown PD, Ballman KV, Cerhan JH, et al. Postoperative stereotactic radiosurgery compared with whole brain radiotherapy for resected metastatic brain disease (NCCTG N107C/CEC.3): a multicentre, randomised, controlled, phase 3 trial. Lancet Oncol 2017;18:1049-60.

25. Gondi V, Pugh SL, Tome WA, et al. Preservation of memory with conformal avoidance of the hippocampal neural stem-cell compartment during whole-brain radiotherapy for brain metastases (RTOG 0933): a phase II multi-institutional trial. J Clin Oncol 2014;32:3810-6.

26. Bernhardt D, Adeberg S, Bozorgmehr F, et al. Outcome and prognostic factors in patients with brain metastases from small-cell lung cancer treated with whole brain radiotherapy. J Neurooncol 2017;134:205-12 .

27. National Comprehensive Cancer Network (NCCN) Clinical Practice Guidelines in Oncology. Small Cell Lung Cancer. Version 3.2020-February 5, 2020.

28. Guidelines of Chinese Society of Clinical Oncology (CSCO). Primary Lung Cancer, 2019. Available online: https://www.dxy.cn/bbs/newweb/pc/post/41232996

29. Borgelt B, Gelber R, Cramer S, et al. The palliation of brain metastasis: Final results of the first two studies by the Radiation Therapy Oncology Group. Int J Radiat Oncol Biol Phys 1980;6:1-9.

30. Borgelt B, Gelber R, Larson M, et al. Ultra-rapid high dose irradiation schedules for the palliation of brain metastases: Final results of the first two studies by the Radiation Therapy Oncology Group. Int J Radiat Oncol Biol Phys 1981;7:1633-8.

(English Language Editor: A. Kassem) 